\title{
On Riddling and Weak Attractors
}

\author{
Peter Ashwin and John R. Terry \\ Department of Mathematics and Statistics, \\ University of Surrey \\ Guildford GU2 5XH, UK
}

February 3, 2000

\begin{abstract}
We propose general definitions for riddling and partial riddling of a subset $V$ of $\mathbf{R}^{m}$ with nonzero Lebesgue measure and show these properties are invariant for a large class of dynamical systems. We introduce the concept of a weak attractor, a weaker notion than a Milnor attractor and use this to reexamine and classify riddled basins of attractors. We find that basins of attraction can be partially riddled but if this is the case then any partial riddling must be evident near the attractor. We use these concepts to aid classification of bifurcations of attractors from invariant subspaces. In particular, our weak attractor is a generalization of the absorbing area investigated
\end{abstract}


by other authors and we suggest that a transition of a basin to riddling

is usually associated with loss of stability of a weak attractor.

Pacs: 05.45.-a 05.45.Xt

Keywords: Riddling, Riddled Basin, Invariant Manifold, Chaotic Dynamical System, Milnor Attractor.

\section{Introduction}

Progress in finite dimensional dynamical systems has been made typically by ignoring information contained in the trajectories. For example, the study of asymptotic properties of trajectories has given rise to a generic qualitative theory that can describe many possible types of asymptotic motion. If the asymptotic motion is chaotic, invariant measures can be used to describe 'typical' asymptotic behaviour by ignoring initial conditions that are insignificant in that they have small measure.

An important development of this type was the introduction by Milnor of a notion of attractor that ignores initial conditions on a set of zero measure [11]. This gave a theoretical framework for understanding attractors with 'riddled basins' [1] that are attracting for a large measure set of initial conditions (basin) but where this basin may have an open dense complement in the phase space. In this paper, we present some definitions and basic results about what we call riddled subsets of $\mathbf{R}^{m}$ and then apply these to describe 
properties of attractors for maps of $\mathbf{R}^{m}$.

In Section 2 we define the riddled component of a subset of $\mathbf{R}^{m}$ with positive Lebesgue area, and in this way generalize the notion of riddling discussed by $[1,3]$. We allow for the possibilities of a subset to be fully, partially or unriddled, and in Theorem 2.1 we give some basic properties of measurable sets in terms of riddling. We show in Theorem 2.2 that the riddled and unriddled components of an invariant set are preserved in a natural way for a wide class of continuous maps satisfying what we refer to as property $(\mathrm{P})$.

Section 3 discusses definitions and examples of attractors for such maps, and we introduce a weaker notion of attractor than Milnor, a weak attractor that we use to discuss synchronization in the final section. In Theorem 3.2 we show that in the case of a partially riddled basin, an attractor must be contained in the (closure) of the riddled and unriddled components of the basin. Theorem 3.3 gives necessary conditions for a Milnor attractor to have a locally but not globally riddled basin. The section ends with examples of a map of $\mathbf{R}^{2}$ with a partially riddled basin, and an invertible map of $\mathbf{R}^{3}$ with a riddled basin.

Section 4 uses these ideas to generalize the notion of absorbing area of Mira and co-workers. We suggest that absorbing areas are special cases of absorbing regions that can be defined for invertible or non-invertible maps or flows in arbitrary finite dimension, without reference to critical curves. 
We use ideas of weak attractors and absorbing regions to discuss some cases where bifurcation can lead to creation of riddled basins of attraction.

\section{Properties of riddled subsets of $\mathbf{R}^{m}$.}

We first fix some notation used throughout the paper. We assume that $M=\mathbf{R}^{m}, \ell($.$) denotes Lebesgue measure on M$, and subsets will be assumed to be Borel subsets of $M$. We denote the $\delta$-ball around $x$ by

$$
B_{\delta}(x)=\{y:|x-y|<\delta\}
$$

In applications of dynamical systems, one is usually only interested in the fate of points up to any subsets of measure zero. To this end we define equality and containment up to zero measure. If $U$ and $V$ are two (Borel) subsets of $M$ we say $U={ }_{0} V$ if $\ell(U \Delta V)=0$, where $U \Delta V=(U \backslash V) \cup(V \backslash U)$ is the symmetric difference. In other words, we say $U={ }_{0} V$ if $U$ and $V$ differ only on a set of measure zero. We denote the complement $V^{c}=M \backslash V$. If $A$ is such that there is an open set $C$ with $A={ }_{0} C$ then we say $A$ is almost open. Similarly, if $A={ }_{0} \emptyset$ we say $A$ is almost empty. (NB we use the convention that $\ell(V)>0$ includes the case $\ell(V)=\infty$.) The following definitions generalize the concept of riddling of Alexander et al. [1] to arbitrary sets.

\section{Definition 2.1}


1. Suppose that $V$ is a measurable subset of $M$. We define the riddled component of $V$ to be

$$
V_{\text {rid }}=\left\{x \in V: \ell\left(B_{\delta}(x) \cap V\right) \ell\left(B_{\delta}(x) \cap V^{c}\right)>0 \text { for all } \delta>0\right\}
$$

2. We refer to $V_{\text {unrid }}=V \backslash V_{\text {rid }}$ as the unriddled component of $\mathrm{V}$.

3. If $V_{\text {rid }}={ }_{0} V \neq 0 \emptyset$ then we say that the set $V$ is (fully) riddled. If $V_{\text {rid }}={ }_{0} \emptyset$ we say it is unriddled. If it is neither riddled nor unriddled, we say it is partially riddled.

4. If $U$ and $V$ have $\ell(U)>0, \ell(V)>0$ and $U \cap V=\emptyset$, and for almost all $x \in U$ and all $\delta>0$ we have

$$
\ell\left(B_{\delta}(x) \cap V\right) \ell\left(B_{\delta}(x) \cap U\right)>0
$$

then we say that $U$ is riddled with $V$.

5. If $U$ is riddled with $V$ and $V$ is riddled with $U$ then we say the sets are intermingled.

Any embedded manifold is unriddled, as is any set with zero Lebesgue measure. A simple example of a riddled set can be constructed as follows: Take a sequence of points $p_{i}$ dense in $[0,1]$ and consider

$$
S_{\epsilon}=[0,1] \backslash\left(\bigcup_{i \in \mathbf{N}}\left[p_{i}, p_{i}+\frac{\epsilon}{2^{i}}\right]\right)
$$

One can show that $\ell\left(S_{\epsilon}\right) \geq 1-\epsilon$ and so if $\epsilon<1$, this set will be fully riddled but still have open dense complement. 
Theorem 2.1 Suppose that $V \neq_{0} \emptyset$ is a measurable subset of $M$.

(i) $V_{\text {rid }}$ is closed in the subset topology of $V$.

(ii) $V_{\text {unrid }}$ is almost open.

(iii) If $V$ is the closure of its interior then $V_{\text {unrid }}=\operatorname{Int}(V)$ and $V_{\text {rid }}=\partial V$.

Proof: (i) Suppose $x_{n}$ is a sequence in $V_{\text {rid }}$ that converges to some point $x \in V$. Consider any $\delta>0$ and note that there is an $N$ and $\epsilon>0$ such that $B_{\epsilon}\left(x_{N}\right) \subseteq B_{\delta}(x)$. Therefore if $U=V$ or $V^{c}$ then

$$
\ell\left(B_{\delta}(x) \cap U\right) \geq \ell\left(B_{\epsilon}\left(x_{N}\right) \cap U\right)>0
$$

and so $x \in V_{\text {rid }}$.

(ii) Let

$$
U=\left\{x \in M: \text { There is a } \delta>0 \text { with } \ell\left(B_{\delta}(x) \cap V^{c}\right)=0\right\}
$$

and

$$
\hat{V}=\left\{x \in V: \text { There is a } \delta>0 \text { with } \ell\left(B_{\delta}(x) \cap V^{c}\right)=0\right\}
$$

and note that if $x \in U$ has $\delta>0$ such that $\ell\left(B_{\delta}(x) \cap V^{c}\right)=0$ then for any $y \in B_{\delta / 2}(x), \ell\left(B_{\delta / 2}(y) \cap V^{c}\right)=0$. Hence $y \in U$ and so $U$ is open.

Observe that trivially $V_{\text {unrid }} \subseteq U \cup \hat{V}$ and suppose that $\ell\left(U \backslash V_{\text {unrid }}\right)>0$. An application of the Lebesgue density theorem gives $\ell(\hat{V})=0$ and by further application of the Lebesgue density theorem we can choose an $x \in U$ such 
that $\ell\left(B_{\delta}(x) \cap V^{c}\right)>0$ for all $\delta>0$ which contradicts the assumption $x \in U$. Hence $U={ }_{0} V_{u n r i d}$ and consequently $V_{u n r i d}$ is almost open as required.

(iii) Let $U=\operatorname{Int}(V)$ and by assumption we have $V=\bar{U}$. Clearly $U \subset V_{\text {unrid }}$. By part (i) and the fact that $V$ is closed, the riddled component $V_{\text {rid }}$ must also be closed and so $V_{\text {unrid }}$ must be an open subset of $V$. Since $U$ contains all open subsets of $V$ we conclude that $U=V_{\text {unrid }}$ and $V_{\text {rid }}=\partial V$.

$Q E D$

\subsection{Mappings of riddled sets}

Assume that a map $f: M \rightarrow M$ is continuous. We say that it is of type (P) if the following is true:

(P1) $f$ is almost everywhere a local homeomorphism, i.e. for $\ell$-almost every $x \in M$ there is a neighbourhood $U$ of $x$ such that $f: U \rightarrow f(U)$ is a homeomorphism.

(P2) $\ell(V)>0$ if and only if $\ell(f(V))>0$ for all measurable $V$.

We do not require that the map is invertible; many non-invertible (e.g. piecewise smooth) maps also satisfy (P). If $f$ is almost everywhere a local diffeomorphism then both (P1) and (P2) follow directly. Unless stated otherwise, we shall assume henceforth that the mappings on $M$ considered in this paper are of type (P). If $f(V)={ }_{0} V$ we say $V$ is almost 
invariant under $f$; in the stronger case that $f(V)=V$ we say $V$ is invariant under $f$.

Theorem 2.2 If $V$ is almost invariant under a map $f: M \rightarrow M$ then $V_{\text {rid }}$ and $V_{\text {unrid }}$ are almost invariant. If additionally $f$ is a local homeomorphism and $V$ is invariant then $V_{\text {rid }}$ and $V_{\text {unrid }}$ are invariant.

Proof: By (P1), for almost all $x \in V$ we can find open neighbourhoods $U_{1}$ of $x$ and $U_{2}$ of $f(x)$ such that $f: U_{1} \rightarrow U_{2}$ is a homeomorphism.

Now consider any $\delta>0$ such that $B_{\delta}(x) \subseteq U_{1}$ and $B_{\delta}(f(x)) \subset U_{2}$. By continuity of $f$ on $U_{1}$ we can find an $\epsilon$ with $\delta>\epsilon>0$ such that $f\left(B_{\epsilon}(x)\right) \subseteq$ $B_{\delta}(f(x))$.

Therefore $\ell\left(B_{\delta}(f(x)) \cap V^{c}\right) \geq \ell\left(f\left(B_{\epsilon}(x)\right) \cap V^{c}\right)$. As $f: U_{1} \rightarrow U_{2}$ is invertible we have $f(A \cap B)=f(A) \cap f(B)$ for any $A, B \subseteq U_{1}$. Now $f(V \cap$ $\left.U_{1}\right)=f\left(V \cap U_{1}\right) \cap U_{2}={ }_{0} V \cap U_{2}$ because $V$ is almost invariant. Hence its complement in $U_{1}$ is almost invariant, ie $f\left(V^{c} \cap U_{1}\right)={ }_{0} V^{c} \cap U_{2}$.

Suppose that $\ell\left(B_{\delta}(f(x)) \cap V^{c}\right)=0$ and note that $\ell\left(f\left(B_{\epsilon}(x) \cap V^{c}\right)\right)=$ $\ell\left(f\left(B_{\epsilon}(x)\right) \cap V^{c}\right) \leq \ell\left(B_{\delta}(f(x)) \cap V^{c}\right)$. Therefore $\left.\ell\left(f\left(B_{\epsilon}(x)\right) \cap V^{c}\right)\right)=0$ and by (P2) we have $\ell\left(B_{\epsilon}(x) \cap V^{c}\right)=0$.

Considering $f^{-1}: U_{2} \rightarrow U_{1}$ this is also a homeomorphism that satisfies (P2) and so for any $\delta>0$ such that $\ell\left(B_{\delta}(x) \cap V^{c}\right)=0$ and $B_{\delta}(x) \subseteq U_{1}$ we can find an $\epsilon>0$ such that $\ell\left(B_{\epsilon}\left(f(x) \cap V^{c}\right)\right)=0$. 
Hence, for almost all $x \in V, \ell\left(B_{\delta}(x) \cap V^{c}\right)=0$ for some $\delta>0$ if and only if $\ell\left(B_{\delta}(f(x)) \cap V^{c}\right)=0$ for some $\delta>0$. This is equivalent to saying that $V_{\text {rid }}$ and $V_{\text {unrid }}$ are almost invariant under $f$.

If $f$ is everywhere a local homeomorphism then the above argument can be applied at all points $x \in V$ and we can conclude that $V_{\text {unrid }}$ and $V_{\text {rid }}$ are both invariant.

$Q E D$

\section{Attractors and riddling}

We start by recalling some standard definitions and giving a very weak notion of an attractor. For $f: M \rightarrow M$ and any $x \in M$ we define

$$
\omega(x)=\bigcap_{N>0} \overline{\bigcup_{n>N} f^{n}(x)},
$$

the $\omega$-limit set of $x$. Suppose now that $x^{*}=\left\{x_{-n}^{*}\right\}_{n=0}^{\infty}$ is a backwards trajectory of $f$ through $x$, that is, such that $f\left(x_{-n}^{*}\right)=x_{-n+1}^{*}$ and $x_{0}^{*}=x$. We define

$$
\alpha\left(x^{*}\right)=\bigcap_{N>0} \overline{\bigcup_{n>N} x_{-n}^{*}}
$$

to be the $\alpha$-limit of $x^{*}$ through $x$.

We say a subset $A$ is absorbing if $f(A) \subseteq A$. If $A$ is compact and absorbing then $\omega(x) \subseteq A$ for all $x \in A$. Moreover, $\omega(x)$ is invariant for any $x$. Given 
any compact invariant set $A$ we define its basin of attraction

$$
\mathcal{B}(A)=\{x \in M: \omega(x) \subseteq A\}
$$

and its unstable set

$$
\mathcal{U}(A)=\left\{x \in M: \text { there is an } x^{*} \text { through } x \text { with } \alpha\left(x^{*}\right) \subseteq A\right\}
$$

If $A$ is invariant then $y \in \mathcal{U}(A)$ means that there is a backwards trajectory through $y$ whose $\alpha$-limit is contained in $A$, in particular $A \subseteq \mathcal{U}(A)$. We use the basin of attraction to define the following notions of attractor.

\subsection{Weak attractors}

Definition 3.1 Suppose that $A$ is a compact invariant subset of $M$. If

$$
\ell(\mathcal{B}(A))>0
$$

then we say $A$ is a weak attractor.

Following Milnor [11] (though note that he assumed the sets are closed, we assume additionally compactness for convenience and because of the applications we consider) we define:

\section{Definition 3.2}

1. If $A$ is a weak attractor and any proper compact invariant subset $A^{\prime} \subseteq A$ satisfies

$$
\ell\left(\mathcal{B}(A) \backslash \mathcal{B}\left(A^{\prime}\right)\right)>0
$$


then we say $A$ is a Milnor attractor.

2. If $A$ is a Milnor attractor and any proper compact invariant subset $A^{\prime} \subseteq A$ satisfies

$$
\ell\left(\mathcal{B}\left(A^{\prime}\right)\right)=0
$$

then we say $A$ is a minimal Milnor attractor.

These definitions are successively more restrictive,

Weak attractor $\Leftarrow$ Milnor attractor $\Leftarrow$ minimal Milnor attractor

and there are examples that show that the implications cannot be reversed. Note that any compact invariant set containing a Milnor attractor is a weak attractor. If $A$ is a compact invariant set that has positive Lebesgue measure then it is a weak attractor, but in dissipative systems, weak attractors themselves will typically have zero measure.

Remark 3.1 A natural measure is an ergodic invariant probability measure $\mu$ such that for a positive measure set of $x$ and any continuous $\phi: M \rightarrow \mathbf{R}$ we have

$$
\lim _{n \rightarrow \infty} \frac{1}{n} \sum_{k=0}^{n-1} \phi\left(F^{k}(x)\right)=\int \phi(y) d \mu(y) .
$$

If $\mu$ is a natural measure then the support of $\mu$ is a minimal Milnor attractor.

Suppose that $V$ is a Borel subset of $M$ such that $V$ contains a compact invariant set $A$. We define the basin of $A$ relative to $V$ to be

$$
\mathcal{B}_{V}(A)=\left\{x \in M: \omega(x) \subseteq A \text { and } f^{k}(x) \subseteq V \text { for all } k \in 0,1,2, \ldots\right\}
$$


If $U \supset V \supset A$ then clearly $\mathcal{B}_{U}(A) \supseteq \mathcal{B}_{V}(A)$.

By examining not just the measure of the basin but also the basin topology we get several more concepts of attractor that are increasingly more restrictive than that of Milnor attraction. An almost open basin attractor is a Milnor attractor whose basin is almost open. An open basin attractor is a Milnor attractor whose basin is open. The strongest notion of attraction we consider is the following:

Definition 3.3 If $\mathcal{B}(A)$ is a neighbourhood of $A$ and for all neighbourhoods $V$ of $A$ there is a neighbourhood $U$ of $A$ such that $f^{n}(U) \subset V$ for all $n \in \mathbf{N}$, we say $A$ is asymptotically stable.

An alternative way to put this is as follows:

Lemma 3.1 An invariant set $A$ is an asymptotically stable attractor if and only if for any neighbourhood $V$ of $A, \mathcal{B}_{V}(A)$ is a neighbourhood of $A$.

Proof: Suppose that $A$ is asymptotically stable. Then given any neighbourhood $V$ of $A$ there is a neighbourhood $U$ such that $U \subset \mathcal{B}_{V}(A)$ and so $\mathcal{B}_{V}(A)$ is a neighbourhood. Conversely, if $\mathcal{B}_{V}(A)$ is a neighbourhood for any neighbourhood $V, \mathcal{B}(A) \supset \mathcal{B}_{V}(A)$ is a neighbourhood and $U=\mathcal{B}_{V}(A)$ will satisfy $f^{n}(U) \subset V$ for all $n \in \mathbf{N}$ Q $\quad Q E D$ 
Observe that an asymptotically stable attractor is not necessarily minimal or even a Milnor attractor, but it is a weak attractor. We now prove some elementary results that relate these concepts of attraction. If $X$ is a subset of $M$ that is not almost empty, we define the limit set $\Omega(X)=\overline{\cup_{x \in X} \omega(x)}$ (NB we take the closure since the union is not necessarily closed) and the likely limit set

$$
\Lambda(X)=\bigcap_{Y={ }_{0} X} \Omega(Y)
$$

where we take the intersection over all Borel $Y$ that differ by $X$ on a set of zero measure (this is referred to as $\Lambda(f, X)$ in Milnor's paper [11]). Note that $\Omega(X) \supseteq \Lambda(X)$ and if $X$ is almost empty then $\Lambda(X)$ is empty. Note that $\Omega(X)$ is a weak attractor containing $\Lambda(X)$.

Lemma 1 of Milnor [11] implies that if $X$ is any positive measure compact absorbing subset of $M$ then $\Lambda(X)$ is an attractor that contains all Milnor attractors in $X$, for the restricted map $f_{\mid X}$. Moreover, the same paper shows that if $X$ is any positive measure then $\Lambda(X)$ is a non-empty closed and invariant; if $\Lambda(X)$ is compact then it is a Milnor attractor.

Suppose that $C$ is any positive measure invariant set contained in a compact absorbing set. If $D \subseteq C$ is not almost empty then we have $\Lambda(D) \subseteq \Lambda(C)$ and so $C \subseteq_{0} \mathcal{B}(\Lambda(C))$.

Lemma 3.2 Suppose that $A$ is a weak attractor. Then there is a Milnor 
attractor $A_{m} \subseteq A$ such that

$$
\mathcal{B}(A)={ }_{0} \mathcal{B}\left(A_{m}\right) .
$$

Proof: Let $A_{m}=\Lambda(\mathcal{B}(A))$; note that $A_{m}$ is an attractor with $A_{m} \subseteq A$ and so $\mathcal{B}\left(A_{m}\right) \subseteq \mathcal{B}(A)$. Now consider any positive measure set $C \subseteq_{0} \mathcal{B}(A)$ and note that $\Lambda(C) \subseteq A_{m}$. Hence $C \subseteq_{0} \mathcal{B}\left(A_{m}\right)$ for all $C$ and therefore $\mathcal{B}(A)={ }_{0} \mathcal{B}\left(A_{m}\right)$. Thus, $A_{m}$ is a Milnor attractor.

$Q E D$

Note that weak attractors containing a single common Milnor attractor need not be contained in each other. For example, consider the mapping on $\mathbf{R}$ given by

$$
f(x)=x+x\left(x^{2}-1\right) .
$$

This has two weak attractors $[-1,0]$ and $[0,1]$. However the only Milnor attractor is the point 0 . More generally we can state the following:

Lemma 3.3 Suppose that $A$ is a weak attractor.

(i) If $A_{m}$ is a minimal (Milnor) attractor $A_{m} \subseteq A$ with $B={ }_{0} \mathcal{B}\left(A_{m}\right)$ and $B$ open such that $B \supseteq A$ then $A_{m}$ is the only (Milnor) attractor in $A$.

(ii) If $\mathcal{B}(A)$ is open then there is a Milnor attractor $A_{m} \subseteq A$ with an almost open basin. 
The first part of the previous Lemma follows on noting that $\Lambda(B)=A_{m}$; the second part can be shown by application of Lemma 3.2.

The next result relates the unstable set of an attractor to notions of attraction.

Theorem 3.1 Suppose that $A$ is a weak attractor with non-empty unstable $\operatorname{set} \mathcal{U}(A)$.

(i) If $\mathcal{U}(A)$ is bounded then $\overline{\mathcal{U}(A)}$ is a weak attractor.

(ii) Suppose that $A$ is an asymptotically stable attractor. Then $\mathcal{U}(A)=A$.

\section{Proof:}

(i) This follows because $\overline{\mathcal{U}(A)} \supseteq A$ and so $\mathcal{B}(\overline{\mathcal{U}(A)}) \supseteq \mathcal{B}(A)$. (ii) Suppose that one can find an $x \in \mathcal{U}(A) \backslash A$ and pick any neighbourhood $V$ of $A$ that does not contain $x$. Since there is a backwards trajectory $x^{*}$ such that $\alpha\left(x^{*}\right) \subseteq A$, given any $U$ neighbourhood of $A$ there is a trajectory that starts in $U$ and leaves $V$ after a finite time. This contradicts the assumption of asymptotic stability and so $\mathcal{U}(A)=A$.

$Q E D$

It may also be the case that $\mathcal{U}(A)$ is unbounded or $\mathcal{U}(A)$ may contain other Milnor attractors. Note that if $A_{m}$ is a Milnor attractor then there may be many (possibly infinitely many) weak attractors $A$ with $\mathcal{B}(A)={ }_{0} \mathcal{B}\left(A_{m}\right)$. 


\subsection{Riddling, intermingling and local riddling of basins}

The work of Alexander et al. [1] showed that one can have a minimal Milnor attractor with basins that are riddled in the following sense:

Definition 3.4 $A$ weak attractor $A$ has a globally riddled basin if $\mathcal{B}(A)$ is fully riddled.

It may also be the case that $\mathcal{U}(A)$ is unbounded or $\mathcal{U}(A)$ may contain other Milnor attractors. Any weak attractor contains a Milnor attractor by Lemma 3.2 and so if $A$ is a weak attractor with a riddled basin then the Milnor attractor $A_{m}$ also has a riddled basin. An important consequence of the previous results is the following:

Theorem 3.2 Suppose that $A$ is a minimal Milnor attractor and let $B=$ $\mathcal{B}(A)$. If $B$ is partially riddled then

$$
A \subseteq B_{\text {rid }} \cap \overline{B_{\text {unrid }}}
$$

Proof: Since $B$ is assumed to be partially riddled, both $B_{\text {rid }}$ and $B_{\text {unrid }}$ have positive measure; therefore $\Lambda\left(B_{\text {rid }}\right)$ and $\Lambda\left(B_{\text {unrid }}\right)$ are nonempty. Clearly both are closed subsets of the compact set $\Lambda(B)=A$ and hence they are Milnor attractors. Because $A$ is minimal it is the only attractor in $\Lambda(B)$ and therefore

$$
A=\Lambda\left(B_{\text {rid }}\right)=\Lambda\left(B_{\text {unrid }}\right)
$$


By Theorem 2.2, both $B_{\text {rid }}$ and $B_{\text {unrid }}$ are almost invariant. Since $B_{\text {rid }}$ is closed in $B$ and moreover all $\omega$-limit sets of points in $B_{\text {rid }}$ remain in $B$, we have $\Lambda\left(B_{\text {rid }}\right) \subseteq B_{\text {rid }}$. Therefore $A=\Lambda\left(B_{\text {rid }}\right) \subseteq B_{\text {rid. }}$. Now $B_{\text {unrid }}$ has positive measure and is almost invariant. Almost invariance implies there is a full measure set of points that remain in $B_{\text {unrid }}$ under iteration and so their limits are in $\overline{B_{\text {unrid }}}$, implying that $\Lambda\left(B_{\text {unrid }}\right) \subseteq \overline{B_{\text {unrid }}}$. Hence $A=$ $\Lambda\left(B_{\text {unrid }}\right) \subseteq \overline{B_{\text {unrid }}}$

$Q E D$

Similarly, if $B$ is a basin relative to some open neighbourhood of $A$ we can conclude that $A$ must be in the intersection of the closures of the riddled and unriddled components of $B$.

A consequence of this result is that any attractor with a basin that has full measure in a neighbourhood of the attractor must have an unriddled basin. For example, Figure 1 shows (in black) part of the local basin of attraction of a periodic orbit for the map

$$
f(x, y)=\left(3.88605 x(1-x)-0.3 x y^{2}, 0.545 e^{x} y-y^{3}\right)
$$

consisting of all points that remain within $y \in[0,0.1]$. This is a particular case of a map studied by [4]; the attractor is in the apparently open region of black. Although the basin boundary becomes very convoluted as one moves further away from the immediate neighbourhood of the periodic orbit, Theorem 3.2 implies that the basin is unriddled. For this example, the periodic 
orbit is hyperbolic and so the basin is open, not just almost open.

$$
\text { Figure } 1 \text { goes here }
$$

Definition 3.5 Suppose that $A$ is a weak attractor. We say it has a locally riddled basin if there exists a neighbourhood $V$ of $A$ such that the basin of $A$ relative to $V$, ie $\mathcal{B}_{V}(A)$, is riddled.

This means that an arbitrarily small ball centered around any point of $\mathcal{B}_{V}(A)$ has a set of positive measure which eventually leaves $\mathrm{V}$ under iteration. Clearly a riddled basin is locally riddled, but vice-versa need not apply; it is possible for a locally riddled basin to be almost open.

NB This is a more restrictive assumption than the definition of local riddling in [3], who assumed that there is an open set $V$ and $U(V)=\bigcap_{n=0}^{\infty} f^{n}(V)$ such that

$$
\ell\left(B_{\delta}(x) \cap U(V)^{c}\right)>0
$$

for all $\delta>0$ and all $x \in A$. We require moreover that the above holds for all $x \in U(V)$. This has the advantage that it excludes some undesirable examples, for example the fixed point $\theta=0$ of the ODE $\dot{\theta}=1-\cos \theta(\bmod$ $2 \pi$ ) is not locally riddled according to our definition, though it is according to the previous definition in [3]. 
Lemma 3.4 Suppose that $A$ is a weak attractor such that $\mathcal{B}(A)$ is locally riddled. Then there is a Milnor attractor $A_{m} \subseteq A$ such that $\mathcal{B}\left(A_{m}\right)$ is locally riddled.

Proof: If $A$ is a weak attractor with a locally riddled basin then there exists a neighbourhood $V$ of $A$ such that $\mathcal{B}_{V}(A)$ is riddled. By Lemma 3.2 there is a Milnor attractor $A_{m} \subseteq A$ such that $V$ is also a neighbourhood of $A_{m}$ and $\mathcal{B}\left(A_{m}\right)={ }_{0} \mathcal{B}(A)$. Hence $\mathcal{B}_{V}\left(A_{m}\right)={ }_{0} \mathcal{B}_{V}(A)$ and consequently $\mathcal{B}_{V}\left(A_{m}\right)$ is riddled.

$Q E D$

It is interesting to note that the converse of Lemma 3.4 is false. In general it is not true that the basin of a weak attractor $A$ is locally riddled if the basin of a Milnor attractor $A_{m} \subseteq A$ is locally riddled.

If the basin is almost open then for almost all $x \in \mathcal{B}(A)$

$$
\ell\left(B_{\delta}(x) \cap \mathcal{B}(A)\right) \ell\left(B_{\delta}(x) \cap \mathcal{B}(A)^{c}\right)=0
$$

for small enough $\delta$. Hence $\mathcal{B}(A)$ is not globally riddled.

Lemma 3.5 Suppose that a weak attractor $A$ has a basin that is almost open. Then its basin is not globally riddled.

This does not however preclude $A$ from having a locally riddled basin even if it is the unique Milnor attractor inside a weak attractor whose basin 
is not locally riddled. The following result gives necessary (but not sufficient) conditions for a basin of an attractor to be locally riddled.

Theorem 3.3 Suppose that $A$ is a weak attractor with an almost open basin and a unique Milnor attractor $A_{m} \subseteq A$. Then $\mathcal{B}\left(A_{m}\right)$ is not globally riddled. If in addition $A_{m} \neq A$ and there is a dense orbit $\left\{f^{n}(\xi)\right\}_{n \in \mathbf{N}}$ in $A$, then the basin of attraction of $A_{m}$ is locally riddled.

Proof: Suppose that $A_{m}$ is the unique Milnor attractor such that $A_{m} \subset A$ implies that $\mathcal{B}\left(A_{m}\right)={ }_{0} \mathcal{B}(A)$. By Lemma $3.5, \mathcal{B}(A)$ is not globally riddled and hence neither is $\mathcal{B}\left(A_{m}\right)$. Now pick any $y \in A$ and $\epsilon>0$ such that

$$
B_{\epsilon}(y) \bigcap A_{m}=\emptyset
$$

Now choose any $V$, a neighborhood of $A_{m}$ such that

$$
V \bigcap B_{\epsilon}(y)=\emptyset
$$

Consider any $x \in \mathcal{B}_{V}\left(A_{m}\right)$ and $\delta>0$. Due to the existence of a dense orbit, there is an $n_{0}$ such that $f^{n_{0}}(\xi) \in B_{\delta}(x)$. Also there is an $n_{1}>n_{0}$ such that $f^{n_{1}}(\xi) \in B_{\epsilon}(y)$. By continuity of $f$, given any neighbourhood $P$ of $y$, there is a $Q \subset B_{\delta}(x)$ such that $f^{n_{1}-n_{0}}(Q) \subseteq P$. Hence $Q \subseteq U(V)^{c}$. Therefore, $B_{\delta}(x) \bigcap U(V)^{c} \supseteq Q$ implies that

$$
\ell\left(B_{\delta}(x) \bigcap U(V)^{c}\right)>\ell(Q)>0
$$


and so we can conclude that the basin of $A_{m}$ is locally riddled.

If a weak attractor $A$ has a basin that is locally riddled but not globally riddled there may be a weak attractor $C$ with $\mathcal{B}(A)={ }_{0} \mathcal{B}(C)$ such that the basin of $C$ is neither locally nor globally riddled; it may even be asymptotically stable! This could happen in particular if $A$ has a locally riddled basin and

$$
\mathcal{B}(\overline{\mathcal{U}(A)})={ }_{0} \mathcal{B}(A)
$$

\subsection{Example of an attractor with a partially riddled basin}

If we examine the mapping

$$
F(x, y)=(f(x, y), g(x, y))
$$

on $x \in[0,1], y \in[-2,2]$ where $f(x, y)=4 x(1-x)\left(1-0.1 y^{2}\right)$ and

$$
g(x, y)=\left\{\begin{array}{cl}
y & y \geq 1 \\
\exp \left(\sin x-\frac{1}{2}\right) y & 1>y \geq 0 \\
y / 2 & y<0
\end{array} .\right.
$$

Note that $f(x, 0)$ is the full logistic map on $y=0, y=0$ is invariant as $g(x, 0)=0$ and $g$ is continuous but only piecewise smooth in $(x, y)$. Figure 2 illustrates the basin of attraction of and attractor in $[0,1]$ that appears to be 
partially riddled; $y \geq 0$ contains the riddled component of the basin while $y<0$ has the almost open component.

$$
\text { Figure } 2 \text { goes here }
$$

For the map (6) one can prove the assertions above that the basin is partially riddled by observing as follows: one can define upper and lower transverse Lyapunov Exponents (L.E.s) (ie for $y>0$ and $y<0$ ) and these are in general different. The transverse L.E.s for $y>0$ are positive for some periodic measures whereas that for the natural measure is negative. For $y<0$ there is uniform contraction with all L.E.s are equal to $-\log 2$ and so this part of the basin is open. In agreement with Theorem 3.2, the attractor in this example is in the intersection of the riddled and the closure of the unriddled component, even though the map $f$ is discontinuous at $\mathrm{y}=1$.

Note that this map does not have well defined L.E.s on $A$ as the derivative at $A$ is not defined. Also, note that the attractor lies in an invariant manifold that splits the basin into two distinct parts. We believe that this is a necessary condition for partial riddling of a basin of attraction to occur and conjecture that the existence of a partially riddled basin in this case is also due to the lack of smoothness. More precisely, we conjecture the following holds in general: 
Conjecture: A minimal attractor $A$ of a $C^{1+\alpha}$ map $f$ with natural measure $\mu$ cannot have a partially riddled basin if all L.E.s with respect to $\mu$ are non-zero.

There are examples where such attractors have riddled or non-riddled basins [1], and lack of riddling is implied by uniform hyperbolicity of an attractor in a smooth system.

\subsection{Example of an invertible map with a riddled basin}

Although the following example may be obvious to some readers, we find it appropriate to include it at this point. We define a map of $[0,1)^{2} \times \mathbf{R}^{+}$to itself by

$$
F(x, y, z)=(f(x), g(x, y), h(x, z))
$$

where $f(x)=2 x-\lfloor 2 x\rfloor, g(x, y)=(y+\lfloor 2 x\rfloor) / 2$ and

$$
h(x, z)=\int_{0}^{z} H(x, z) d z
$$

where $H(x, z)$ a positive function, smooth in $x$ and $z$ that satisfies $H(x, 0)=$ $\exp \left(\frac{1}{3}-x\right), H(x, z) \rightarrow 2$ as $z \rightarrow \infty$ (uniformly in $x$ ) and which is monotonic increasing in $z>0$ for any fixed $x$.

This map on $[0,1)^{2} \times \mathbf{R}^{+}$is invertible (the map on $(x, y)$ is the Baker's transformation and $z \rightarrow h(x, z)$ is invertible due to positivity of $H$ ). The natural measure for the Baker's map is simply two dimensional Lebesgue 
measure on $[0,1)^{2}$ and the mapping leaves the subspace $z=0$ invariant. There is also an attracting fixed point at $z= \pm \infty$. One can compute the transverse L.E. for any ergodic invariant measure $\mu$ in $z=0$ as

$$
\begin{aligned}
\Lambda(\mu) & =\int \log \left|\frac{\partial h}{\partial z}\right| d \mu(x, y), \\
& =\int \log |H(x, 0)| d \mu(x, y), \\
& =\int \log \left|\exp \left(\frac{1}{3}-x\right)\right| d \mu(x, y), \\
& =\int\left(\frac{1}{3}-x\right) d \mu(x, y) .
\end{aligned}
$$

For the natural measure this is $\Lambda\left(\mu_{n}\right)=-\frac{1}{6}<0$ but for the fixed point at $(x, y)=(0,0)$ is $\Lambda=\frac{1}{3}>0$. Note moreover that points of the form $(0,0, z)$ with $z \neq 0$ will iterate to infinity. Since there is a dense set of points that are asymptotic to the fixed point $(0,0)$, we can conclude that the plane $z=0$ is an attractor with a riddled basin (see e.g. $[1,3]$ ).

\section{Absorbing regions}

The concept of an absorbing area of a two dimensional non-invertible map was introduced in 1977 [6] as a region in $\mathbf{R}^{2}$ bounded by images of critical curves. This has proven very useful in describing the structure of attractors for two dimensional non-invertible maps. More recently, a number of articles $[5,7,9,10]$ have shown that there are connections between absorbing areas and the bifurcations and basins of attraction of systems exhibiting riddled 
basins and blowout bifurcations.

We generalise the idea of an absorbing area to consider absorbing regions; these can be defined even for smooth invertible maps that do not have critical curves in the sense of [6], and hence we should also be able to extend these ideas to apply to flows generated by ODEs. As before we suppose that $M=\mathbf{R}^{m}$, and that we have a type $(\mathrm{P})$ map,

$$
f: M \rightarrow M
$$

Definition 4.1 We say that an unriddled set $A \subseteq M$ is an absorbing region for $f$ if the following properties are satisfied:

(A1) $f(A) \subseteq_{0} A$.

(A2) $\ell(A)>0$.

Note the fact that $A$ is unriddled implies that $A$ is almost open, (A2) implies that $A \neq \neq_{0} \emptyset$.

An absorbing area in the sense of $[5,6,9]$ is an absorbing region in our sense but they also require that $A$ has a boundary that consists of segments of images of a critical curve corresponding for differentiable maps to $L C=$ $\{x \in M: \operatorname{det}(D f(x))=0\}$. Our absorbing region is a generalisation in that the map $f$ can be invertible and/or smooth, and the notion generalises easily to flows or semiflows in arbitrary dimension as long as the time- $T$ maps satisfy property $(\mathrm{P})$. The phase space is not restricted to one or two dimensions. 
The strength of using critical curves is that they provide a tool one can use to construct absorbing regions numerically. We do not suggest that absorbing areas are in general easy to find, nor do we aim to give any methods to find them.

If $\mathcal{A}$ is an absorbing region such that $f(\mathcal{A})=\mathcal{A}$ we say it is an invariant absorbing region. Noting that $\ell(\mathcal{B}(\mathcal{A})) \geq \ell(\mathcal{A})>0$ leads us to conclude the following:

Lemma 4.1 If $\mathcal{A}$ is an invariant absorbing region then it is a weak attractor.

In other words, an invariant absorbing region is a special case of a weak attractor. More generally, if an absorbing region is not invariant it must contain a weak attractor which can be constructed by

$$
A=\bigcap_{n \in \mathbf{N}} f^{n}(\mathcal{A})
$$

along the lines of $[11$, Lemma 1].

In [9], Maistrenko et al. consider a system of two coupled logistic maps and demonstrate how a synchronized chaotic attractor can lose stability via either a hard or soft blowout bifurcation. They give evidence that the change from hard to soft blowout is caused by a collision between the basin boundary and the absorbing area $\mathcal{A}$ containing the attractor. More recently, Bischi and Gardini [5] show that this can only be the case if $\mathcal{A}$ is an invariant absorbing area, as an absorbing area for which $f(\mathcal{A}) \subseteq \mathcal{A}$ is a proper containment will 
have transient points on the boundary. Moreover they indicate that their arguments in [5] go through if one considers absorbing area of mixed type (these have boundaries that consist not only of segments of critical curves). We indicate here that their results can be understood in the general setting of weak attractors; the precise form of the boundary of a weak attractor appears to be unimportant.

\subsection{Absorbing regions and bifurcations of chaotic at- tractors}

Suppose we have a family of type $(\mathrm{P})$ mappings

$$
f_{r}: M \rightarrow M
$$

indexed by $r \in \mathbf{R}$ such that $f$ changes continuously with $r$. Suppose that $f_{r}$ has a continuous family of invariant absorbing regions $\mathcal{A}_{r}$ (recall that these are weak attractors) and a family of minimal Milnor attractors $A_{r} \subseteq \mathcal{A}_{r}$ such that

- $A_{r} \neq_{0} \mathcal{A}_{r}$ for $r<r_{0}$

- $A_{r}={ }_{0} \mathcal{A}_{r}$ for $r>r_{0}$.

Then we say $A_{r}$ undergoes an expansion bifurcation at $r=r_{0}$. The following is a simple consequence of Theorem 3.3. 
Lemma 4.2 Suppose $f_{r}$ has a family of attractors that undergoes an expansion bifurcation at $r_{0}$. Then no $A_{r}$ has a globally riddled basin.

This has the following consequence for blowout bifurcations [13]: suppose that an attractor inside an almost open invariant absorbing region undergoes a blowout such that at all times there is only one attractor within the absorbing region. Then this blowout must be supercritical [2].

Note that a weak attractor can undergo a bifurcation itself without the attractor within bifurcating. Such a bifurcation can take the form of a crisis where the boundary of a weak attractor hits some unstable dynamics that is not in its basin of attraction. This will typically lead to destruction of the weak attractor.

If $\mathcal{A}$ is an invariant absorbing region that is asymptotically stable, then $\mathcal{A}$ will persist under small perturbations of the map. Correspondingly, if an absorbing region suddenly ceases to exist, this must be due to its basin of attraction (which is almost equal to the basin of attraction of Milnor attractors inside the region) touching the boundary of the absorbing region.

We say a branch of minimal Milnor attractors $A_{r}$ (that may or may not be contained in absorbing areas) undergoes a bifurcation to (global) riddling if there are basins of attraction $B_{r}=\mathcal{B}\left(A_{r}\right)$ such that

- $B_{r}$ is unriddled for $r<r_{0}$.

- $B_{r}$ is riddled for $r>r_{0}$. 
Similarly, we say there is a bifurcation to local riddling if $A_{r}$ is contained in a family of neighbourhood $U_{r}$ and the above holds for $B_{r}=\mathcal{B}_{U_{r}}\left(A_{r}\right)$. In the literature, there are several ways that global riddling can happen, and the above definition brings the following cases together:

1. An attractor in an invariant subspace can lose asymptotic stability at what is called a riddling bifurcation, or a bifurcation to bubbling $[2,8,12,18]$. In this case unstable dynamics bifurcates away from $A_{r}$ transversely, typically a periodic orbit loses transverse stability.

2. The basin of attraction of an attractor can undergo a crisis leading to 'leakage' from the basin. This will occur if the attractor is contained inside a weak attractor $\mathcal{A}_{r}$ that loses asymptotic stability; for example if the invariant absorbing region has a boundary crisis and hits part of its basin boundary. This case has been investigated recently by Maistrenko et al. $[7,9,10]$ and gives a transition from local riddling to global riddling.

3. A chaotic attractor in an invariant subspace is created at bifurcation from a stable periodic orbit. This situation was found in [4] to occur readily in systems with parameters that do not preserve dynamics on invariant subspaces (non-normal parameters).

These three cases above are shown schematically in Figure 3. It is quite possible that there are other mechanisms by which these bifurcations may 
occur and there is still evidently much work needed before we can say that we understand generic bifurcations to riddling in chaotic systems.

\section{Figure 3 goes here}

\section{Discussion}

In this paper we have tried to understand some of the interplay between topology and measure in dynamical systems that possess invariant sets that are riddled, with the aim of understanding the appearance and structure of basins that have a riddled component. A more precise quantitative classification of riddled sets using, for example, exterior dimension should also be possible but we have not considered this up to now. We have also not considered the implications for the dynamics on basin boundaries and expect that bifurcations to riddling will affect this greatly; see for example [16]

By introducing the concept of a weak attractor, we have demonstrated sufficient conditions to ensure that a basin of attraction is or is not globally riddled, a fact which has important consequences in particular for synchronization problems $[2,14,15,17]$. For example, if two or more oscillators are coupled in a way that preserves a symmetry, riddled basins have been observed to appear in a very natural way. Indeed, they have not been seen robustly in systems that do not have invariant submanifolds. The existence of riddled basins is associated with instability of an attractor to perturbations 
by noise and so is of important interest in applications.

\section{Acknowledgements}

We gratefully acknowledge very interesting conversations with Yuri Maistrenko and Leonid Bunimovich, and also the referees for some very helpful comments and pointing out some errors in an earlier version of the manuscript. PA and JT would like to thank the EPSRC for support via grant GR/K77365.

\section{References}

[1] Alexander, J.C., Yorke, J.A. You, Z. and Kan, I. Riddled Basins. Int. J. Bif. Chaos 2, 795-813 (1992).

[2] Ashwin, P., Buescu, J. and Stewart, I., Bubbling of attractors and synchronisation of oscillators. Phys. Lett. A 193, 126-139 (1994).

[3] Ashwin, P., Buescu, J. and Stewart, I., From attractor to chaotic saddle: a tale of transverse instability. Nonlinearity 9, 703-737 (1996).

[4] Ashwin, P., Covas, E. and Tavakol, R., Transverse instability for nonnormal parameters. Nonlinearity 12, 563-577 (1999).

[5] Bischi, G.-I. and Gardini, L., Role of invariant and minimal absorbing areas in chaos synchronization. Phys Rev E 58, 5710-5719 (1998). 
[6] Gumowski, I. and Mira, C., Comptes Rendus Acad. Sc. Paris, 285, 477-480 (1977).

[7] Kapitaniak, T., Maistrenko, Yu., Stefanski, A. and Brindley, J., Bifurcations from locally to globally riddled basins. Phys. Rev. E 57, R6253-R6256 (1998).

[8] Lai, Y-C., Grebogi, C., Yorke, J.A., and Venkataramani, S.C., Riddling bifurcation in chaotic dynamical systems. Phys. Rev. Lett. 77, 55-58 (1996)

[9] Maistrenko, Yu. L., Maistrenko, V. L., Popovich, A. and Mosekilde, E., Role of the absorbing area in chaotic synchronization. Phys. Rev. Lett. 80, 1638-1641 (1998).

[10] Maistrenko, Yu. L., Maistrenko, V.L., Popovich, A. and Mosekilde, E., Unfolding the riddling bifurcation, Preprint, (1998).

[11] Milnor, J., On the concept of attractor. Commun. Math. Phys. 99, 177195 (1985); Comments Commun. Math. Phys. 102, 517-519 (1985).

[12] Ott, E., Sommerer, J.C., Alexander, J., Kan, I. and Yorke, J.A. A transition to chaotic attractors with riddled basins. Physica D 76, 384410 (1994). 
[13] Ott, E. and Sommerer, J. Blowout bifurcations: the occurrence of riddled basins and on-off intermittency. Phys. Lett. A 188, 39-47 (1994).

[14] Pikovsky, A.S., On the interaction of strange attractors. Z. Phys. B, 55, 149-154, (1984).

[15] Pikovsky, A.S. and Grassberger, P., Symmetry breaking of coupled chaotic attractors. J. Phys. A, 24, 4587-4597, 1991.

[16] Rosa Jr., E. and Ott, E. Mixed basin boundary structures of chaotic systems. Phys. Rev. E 59, 343-352 (1999).

[17] Yamada, H. and Fujisaka, T.. Stability theory of synchronised motion in coupled-oscillator systems. Prog. Theor. Phys. 70, 1240-1248 (1984).

[18] Venkataramani, S.C., Hunt, B.R., Ott, E., Gauthier, D.J. and Bienfang, J.C. Transitions to bubbling of chaotic systems. Phys. Rev. Lett. 77, $5361-5364(1996)$. 


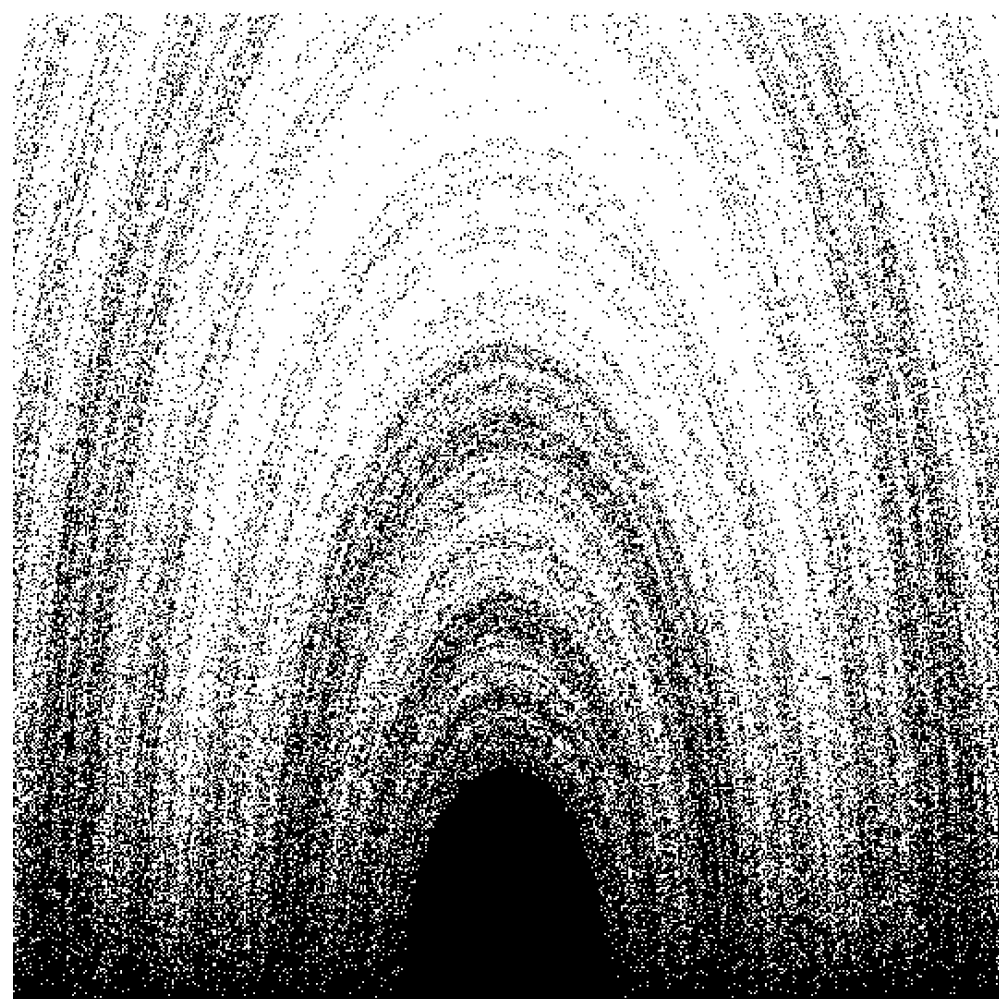

Figure 1: The black region shows that computed basin of attraction of an attractor for the map (4) relative to the strip $0 \leq y \leq 0.1$. The basin in the area $(x, y) \in[0.49,0.50] \times[0 ., 0.05]$ is shown. Observe that the (local) basin contains open sets and these contain a stable attracting periodic point in $y=0$. As a result of Theorem 3.2 we can conclude that the basin is totally unriddled. (Reproduced from [4]). 


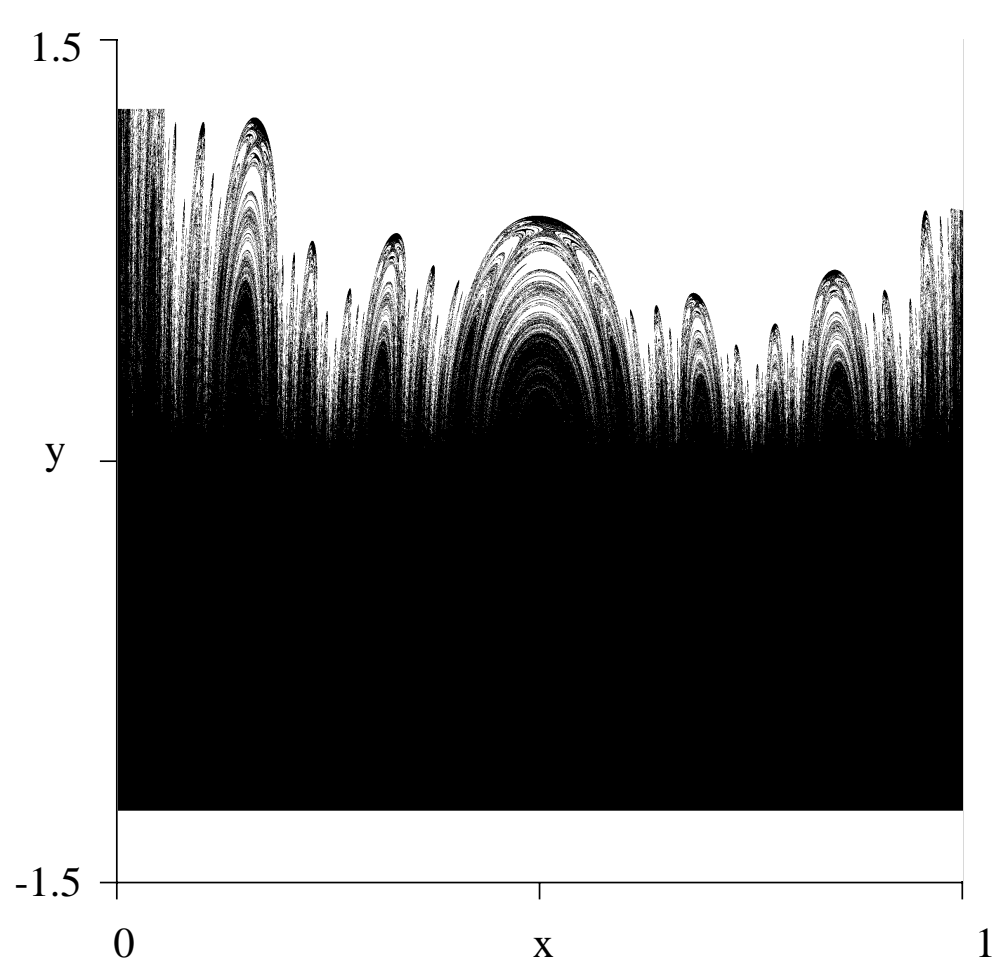

Figure 2: The black region shows a numerical approximation of a partially riddled basin of attraction for an attractor of the map (6) that lies in $y=0$. Observe that the basin has a riddled component in $y>0$ and unriddled component in $y<0$. In agreement with Theorem 3.2, the attractor lies in the intersection of the closures of the riddled and unriddled components. 


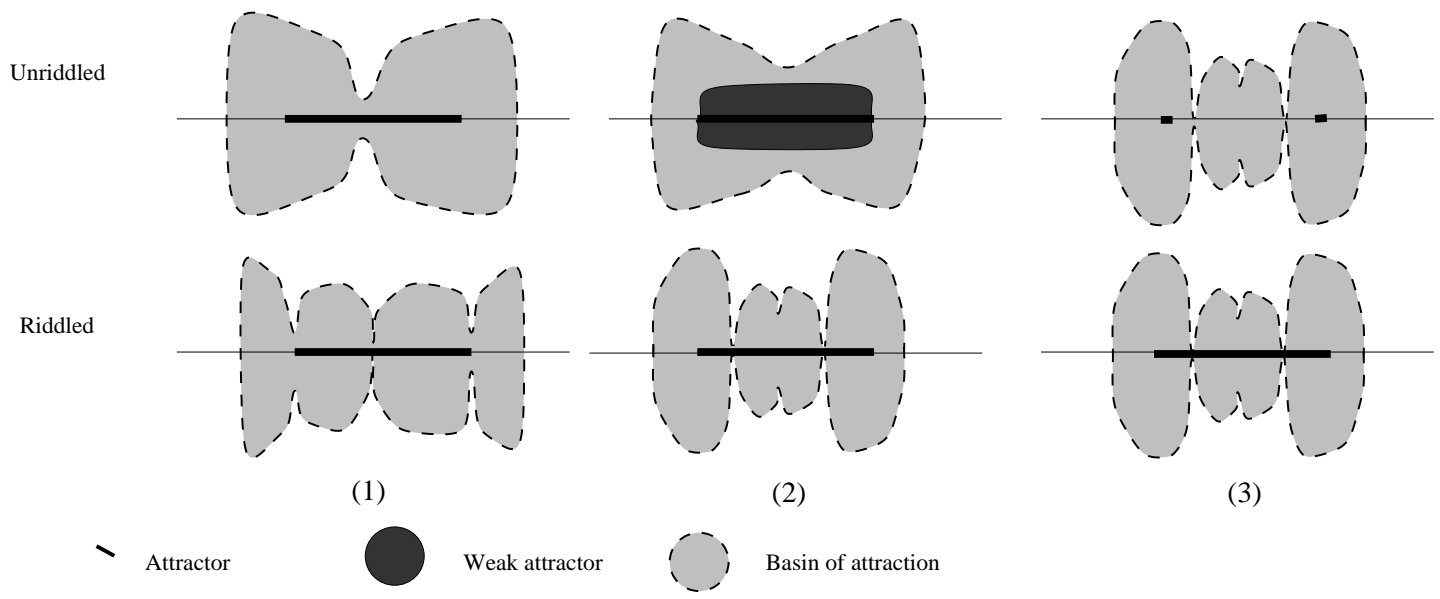

Figure 3: The three cases of bifurcation to global riddling of basins as discussed in the text. In all cases there is an attractor contained in the horizontal invariant subspace. Points outside the grey region are assumed to be in the basin of some other attractor. In case (1) an attractor in an invariant subspace loses asymptotic stability via a transverse bifurcation. In case (2) an attractor is contained in a weak attractor and the latter loses asymptotic stability at a crisis. In case (3) an attractor in an invariant subspace undergoes an explosion which causes it to lose asymptotic stability. In case (2) note that although the weak attractor is destroyed at a crisis with the basin boundary, many invariant sets within the weak attractor will persist on the basin boundary. Case (3) requires perturbation within the invariant subspace but cases (1) and (2) do not. Case (2) has a locally riddled basin before the bifurcation whereas (1) and (3) do not. 